\title{
Molecular beam epitaxy growth of MgZnSSe/ZnSSe Bragg mirrors controlled by in situ optical reflectometry
}

\author{
P. Uusimaa, K. Rakennus, A. Salokatve, and M. Pessa \\ Department of Physics, Tampere University of Technology, P.O. Box 692, FIN-33101 Tampere, Finland
}

T. Aherne, J. P. Doran, J. O'Gorman, and J. Hegarty

Department of Physics, Trinity College, Dublin 2, Ireland

(Received 23 June 1995; accepted for publication 6 August 1995)

\begin{abstract}
In situ optical reflectometry at the wavelength of $488 \mathrm{~nm}$ was employed to control the growth of $\mathrm{MgZnSSe} / \mathrm{ZnSSe}$ Bragg mirror stacks for the blue-green spectral region. 10- and 20-period layer structures of $\mathrm{MgZnSSe} / \mathrm{ZnSSe}$ were grown on GaAs (100) epilayers by molecular beam epitaxy. A room-temperature peak reflectance of $86 \%$ was obtained for the 20 -period structure at the central wavelength of $474 \mathrm{~nm}$. The results show that, in general, in situ optical monitoring of growth is a viable and simple method for real-time layer thickness control of $\mathrm{MgZnSSe} / \mathrm{ZnSSe}$ quarter-wave stacks. (C) 1995 American Institute of Physics.
\end{abstract}

Semiconductor microcavities are being exploited in novel optoelectronic devices such as vertical cavity surface emitting lasers (VCSELs) and high efficiency planar surface light emitting diodes. Microcavities may also be used for exploring fundamental properties of the coupling of electromagnetic radiation and two-dimensional electronic excitations in quantum wells, i.e., cavity polaritons. ${ }^{1}$ In all of these applications high reflectance mirrors are needed.

Within the family of ZnSe-based II-VI semiconductors, which are of great importance for the blue-green spectral region, vertical cavity structures have only been realized by means of an epitaxial lift-off (ELO) method $^{2}$ or by selective etching ${ }^{3}$ followed by deposition of dielectric or metallic mirrors on both sides of the cavity. However, growing an epitaxial II-VI semiconductor distributed Bragg reflector (DBR) directly into the layer structure is a more desirable possibility. This approach is very attractive because there are many problems encountered in the ELO and selective etching methods.

We have already demonstrated a high reflectance $\mathrm{MgZnSSe} / \mathrm{ZnSSe}$ quarter-wave stack grown by molecular beam epitaxy (MBE), ${ }^{4}$ which proved the feasibility of fabrication of Bragg mirror stacks from ZnSe-based materials. It was found, however, that MnZnSSe is more difficult to dope $p$ type than MgZnSSe. In addition, MgZnSSe has a larger fundamental band gap than MnZnSSe and, consequently, a smaller refractive index $(n)$. Therefore, we began to study $\mathrm{MgZnSSe} / \mathrm{ZnSSe}$ as a new candidate for high reflectance DBR mirrors intended for II-VI devices.

Fabrication of high reflectance MgZnSSe/ZnSSe Bragg mirrors is challenging because of structural instabilities, such as compositional variations, which are involved in the growth of ZnSe-based compound semiconductors. We have employed an in situ laser reflectometry method (see, e.g., Ref. 5) during growth of $\mathrm{MgZnSSe} / \mathrm{ZnSSe} \mathrm{DBRs}$ in order to determine accurately and straight forwardly the $\lambda / 4 n$ layer thicknesses at growth temperature. We used an air cooled argon ion laser emitting at $\lambda=488 \mathrm{~nm}$. Proper layer thicknesses can be achieved by switching the growth of materials at the extrema of reflected laser beam intensity. In this way the difficulties related to growth rate and compositional variations can be overcome to a large extent.

$\mathrm{MgZnSSe}$ has been widely used as a cladding layer of blue-green laser diodes. ${ }^{6,7}$ The room-temperature band gap $\left(E_{g}\right)$ of $\mathrm{MgZnSSe}$ lattice matched to GaAs can be varied from 2.68 to about $3.9 \mathrm{eV}$ by increasing the contents of sulphur and magnesium. Increasing $E_{g}$ reduces $n^{8}$ and makes $\mathrm{MgZnSSe}$ suitable as low-index layers of the quarter-wave stacks. One of the major obstacles for preparing such layer stacks from $\mathrm{MgZnSSe} / \mathrm{ZnSSe}$ is the increasing instability in MBE growth of MgZnSSe with high sulphur concentrations. ${ }^{9}$ This instability together with the difficulties in $p$-type doping lead to a practical lower limit of $n \cong 2.5$ in the wavelength range of interest $(460-500 \mathrm{~nm})$. Due to a small refractive index difference $(\Delta n)$, which is 0.3 at most, the preparation of high reflectance $\mathrm{MgZnSSe} / \mathrm{ZnSSe}$ Bragg mirrors remains a challenge. But our theoretical calculations based on transfer matrix method indicate (Fig. 1) that $\mathrm{MgZnSSe} / \mathrm{ZnSSe}$ could be suitable for VCSEL structures which require high mirror reflectivities, typically $R>99 \%$.

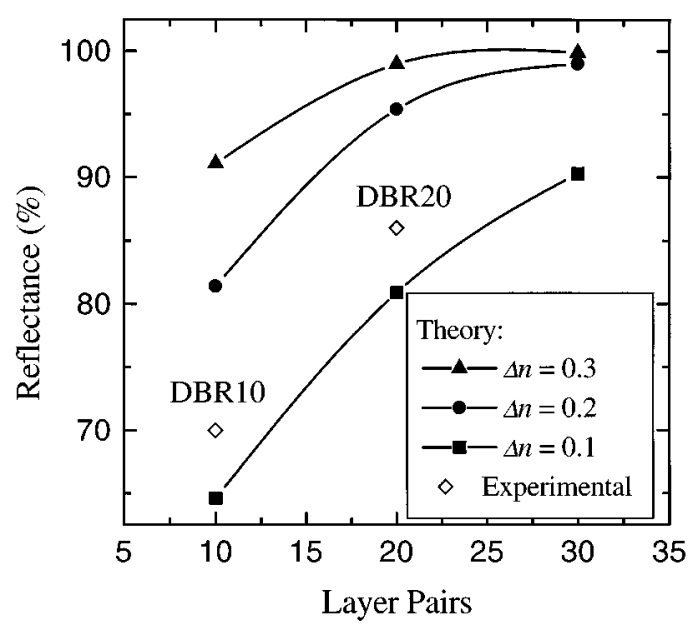

FIG. 1. A theoretical study (transfer matrix method) of the maximum reflectance for 10-, 20-, and 30-period $\mathrm{MgZnSSe} / \mathrm{ZnSSe}$ DBRs with several $\Delta n$ values $\left(n_{\mathrm{ZnSSe}}=2.8\right)$. Experimental reflectances for DBR10 and DBR20 are also shown. 
To obtain this $R=99 \%$ with a reasonable $\Delta n=0.2$, one needs 30 pairs of $\mathrm{Mg}_{0.14} \mathrm{Zn}_{0.86} \mathrm{~S}_{0.20} \mathrm{Se}_{0.80} / \mathrm{ZnS}_{0.07} \mathrm{Se}_{0.93}$.

In this letter we present for the first time the fabrication and optical and structural characteristics of 10- and 20-period $\mathrm{MgZnSSe} / \mathrm{ZnSSe}$ Bragg mirrors. The growth was controlled by in situ optical reflectance measurements.

The mirror structures were grown on GaAs epilayers which were deposited onto GaAs (100) substrates in a III-V MBE system. The GaAs layers were protected from air contaminations by $\mathrm{As}_{2}$ coating prior to transferring them into a separate II-VI MBE system. The $\mathrm{As}_{2}$ layer was removed by heating the sample in the II-VI MBE system at $400{ }^{\circ} \mathrm{C}$. Then a thin ZnSe $(\sim 25 \AA)$ buffer layer and the MgZnSSe/ ZnSSe DBR structure were grown. All the II-VI layers were grown at $270{ }^{\circ} \mathrm{C}$. Molecular beam fluxes were measured prior to growth by a nude ionization gauge placed in front of the substrate position. Before preparing the actual mirror structures individual layers of the constituent materials were grown for a rough calibration of growth rates and compositions. Thicknesses of the calibration layers were measured with a stylus profilometer. The composition was determined by ex situ double crystal x-ray diffraction (DCXRD) and room-temperature photoluminescence measurements.

Deposition of the 10- and 20-period layer structures, hereafter referred to as DBR10 and DBR20, was monitored by the $488 \mathrm{~nm}$ laser beam. The laser beam was directed normal to the sample surface through a beam splitter and was modulated by a chopper. The reflected signal was detected using a silicon photodiode and demodulated by a lock-in amplifier. The reflected intensity was recorded by a chart recorder according to which the growth of layer pairs were controlled manually.

In situ reflectance measurement allows a real-time determination of the $\lambda / 4 n$ layer thickness of a constituent material without accurate knowledge of the growth rate or $n$ of the layer. A small drawback of this method is a change of $n$ to a lower value ( $E_{g}$ increases), when the sample is cooled down to room temperature. Fortunately, according to simulations based on the transfer matrix method, this change in $n$ only causes the central maximum of the stop band $\left(\lambda_{0}\right)$ to slightly shift toward a shorter wavelength while peak reflectivity remains almost unchanged. The shift of $\lambda_{0}$ is found to be about $10-15 \mathrm{~nm}$, depending on the compositions of the constituent materials.

Room-temperature reflectance measurements for the DBRs were performed by a double-arm reflectometer ${ }^{4}$ against a commercial mirror exhibiting a certified reflectance of $>98 \%$ over the wavelength range of interest. This setup allows a noise and light source drift independent reflectance measurement with an experimental error $< \pm 1 \%$. Figure 2 shows reflectance spectra for the DBR10 and DBR20 taken at normal incidence. The maximum reflectances were found to be $70 \%$ for DBR10 and $86 \%$ for DBR20 at $473 \mathrm{~nm}$. A comparison of theory and experiment yields $\Delta n \approx 0.12$ $(\Delta n / n \approx 4.3 \%)$ for both of the samples (Fig. 1). So small a $\Delta n$ value leads to a narrow stop-band width. However, reproducibility of the stop-band position in the present measurements is superior to our earlier experiments made without the in situ optical probe. Another difference between the

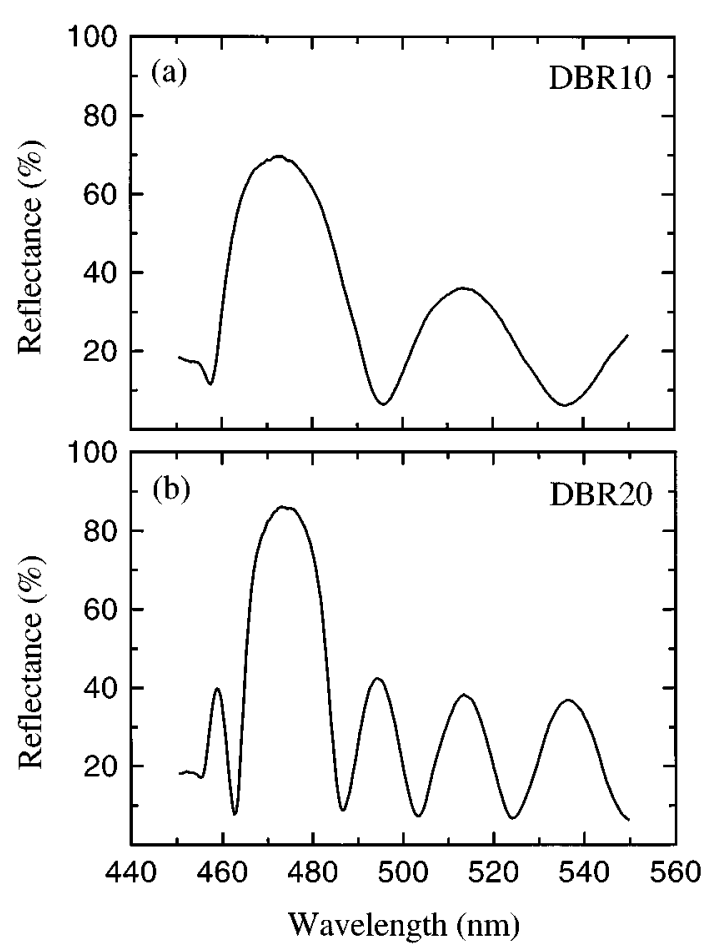

FIG. 2. The reflectance spectrum from a 10-period DBR10 sample (a) and a 20-period DBR20 sample (b) showing maximum reflectances of $70 \%$ and $86 \%$ at $473 \mathrm{~nm}$, respectively.

present and the earlier experiment is that $n$ decreases much more rapidly for $\mathrm{Mg}_{x} \mathrm{Zn}_{1-x} \mathrm{~S}_{y} \mathrm{Se}_{1-y}$ when $\mathrm{S}$ and $\mathrm{Mg}$ are alloyed with $\mathrm{ZnSe}$ compared to $\mathrm{Mn}_{x} \mathrm{Zn}_{1-x} \mathrm{~S}_{y} \mathrm{Se}_{1-y}$. For our earlier 25-period $\mathrm{Mn}_{0.19} \mathrm{Zn}_{0.81} \mathrm{~S}_{0.24} \mathrm{Se}_{0.76} / \mathrm{ZnS}_{0.07} \mathrm{Se}_{0.93}$ we only obtained $\Delta n \approx 0.07$ and $R_{\max }=81 \%$, ${ }^{4}$ while for the DBR20 $\Delta n \approx 0.12$ was achieved with $x=0.15$ and $y=0.18$.

Structural properties of the MgZnSSe/ZnSSe quarterwave stacks were studied by DCXRD (Fig. 3). For the 10period DBR10 remarkable stability of the composition and periodicity was obtained. For the 20-period DBR20 each superlattice diffraction peak exhibits a fine structure. A similar multiple peak pattern is often seen also for a single MgZnSSe layers, and it is caused by a compositional variation in the layer during growth. ${ }^{9}$ Adverse effects of these variations in composition, and in $n$, on the fabrication of high reflectance mirrors can be avoided by using an in situ probe, which measures the effective optical thickness of each layer during the growth. For this purpose the optical reflectance method seems to be very convenient.

The dynamic theory of $\mathrm{x}$-ray diffraction that we used to simulate the $\mathrm{x}$-ray rocking curves resulted in the average period thickness of 95.3 and $93.3 \mathrm{~nm}$ for DBR10 and DBR20, respectively. The experimental spectrum for the DBR20 could be simulated rather accurately by assuming a linear increase of $1.5 \%$ in sulphur concentration of MgZnSSe layers from the first layer to the 15th layer in the stack [Fig. 3(b)]. This increase in sulphur concentration is most probably due to a change in growth temperature which may sometimes decrease as much as $15^{\circ} \mathrm{C}$ during growth of a $0.9-\mu \mathrm{m}$-thick MgZnSSe layer. ${ }^{9}$ In addition, layer thicknesses in the XRD simulation were varied according to the measured 

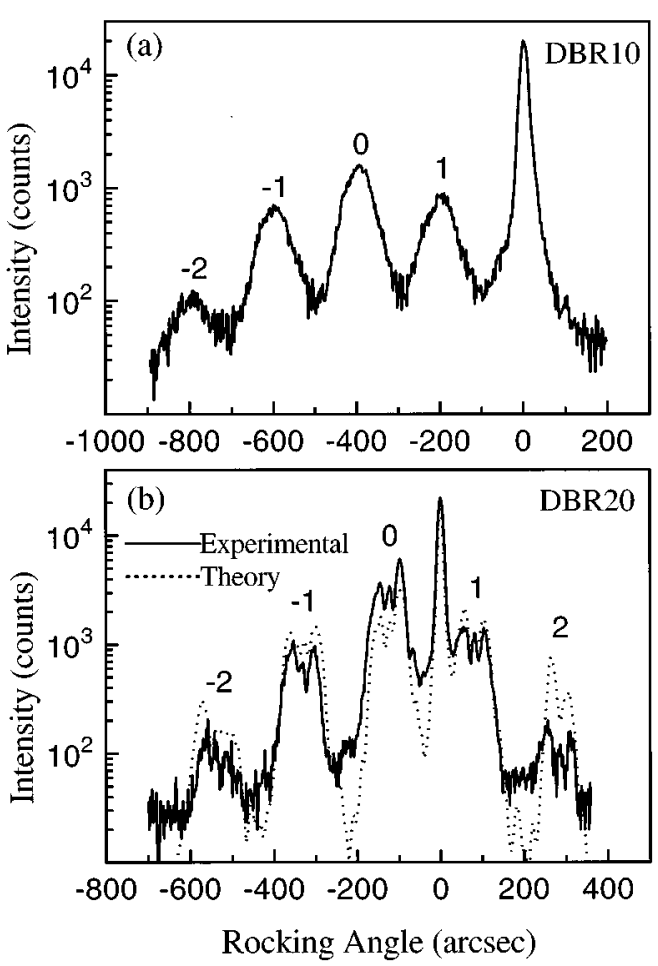

FIG. 3. A DCXRD rocking curve from a 10-period DBR sample (a) and a 20-period DBR20 sample. (b) An XRD simulation (see text) for the DBR20 is also shown.

growth times recorded by the in situ optical reflectance measurement and by assuming a constant average growth rate. The growth rate was calculated from the average period thickness (ex situ DCXRD measurement) and average growth times (in situ reflectance measurement) for DBR20. The standard deviation of layer thickness was $4.3 \%$ for $\mathrm{ZnSSe}$ and $6.1 \%$ for MgZnSSe layers.

The variation of layer thickness (i.e., growth time per layer) was caused by an inaccuracy in determining the maximum reflection point in the reflectance measurement when the number of layer pairs in the stack increased. The result was that the thickness of a ZnSSe layer was not always exactly $\lambda / 4 n$, but varied slightly according to decisions made in manual switching. However, sharp minimum points of the reflection signal could be found easily during growth which allowed an accurate determination of the total thickness of $\lambda / 2 n$ periods. The accurate thickness of the $\lambda / 2 n$ periods was confirmed by summation of the subsequent $\lambda / 4 n$ layers, which showed that the period thickness increased almost linearly from 89.5 to $94.4 \mathrm{~nm}$ during growth. This linear increase can be explained by a decrease of $n$ in MgZnSSe layers due to variation in sulphur concentration. The reflectance simulation showed, however, that the thickness variation has only a minor effect on the reflectance maximum, which is in agreement with an earlier study. ${ }^{10}$

In conclusion, 10- and 20-period $\mathrm{MgZnSSe} / \mathrm{ZnSSe}$ distributed Bragg reflectors at blue-green wavelengths have been grown by MBE and their optical and structural properties have been studied. Instabilities often related to growth of these materials are almost completely surpassed by using in situ optical reflectometry for the measurement of $\lambda / 4 n$ layer thickness during growth. A maximum reflectance of $86 \%$ is obtained for the 20-period reflector. Our results show that the MBE technique combined with optical reflectometry is suitable for direct growth of VCSELs and other microcavity devices which are based on II-VI compound semiconductors. Therefore, the major obstacle for preparing electrically injected $\mathrm{MgZnSSe} / \mathrm{ZnSSe}$ VCSELs is no longer a difficulty in growing these layers, but rather an inherently low $p$-type conductivity of the Bragg mirrors.

This work is carried out within the European ESPRIT Basic Research Project No. 8447 (SMILES). It was also supported, in part, by the Finnish national program EPIMATTER financed by the Academy of Finland and by Optronics Ireland, the Irish national initiative in optoelectronics.

${ }^{1}$ R. Houdre, R. P. Stanley, U. Oesterle, and M. Ilegems, Phys. Rev. B 49, 16761 (1994).

${ }^{2}$ C. Brys, F. Vermaerke, P. Demeester, P. Van Daele, K. Rakennus, A. Salokatve, P. Uusimaa, M. Pessa, A. L. Bradley, J. P. Doran, J. O'Gorman, and J. Hegarty, Appl. Phys. Lett. 66, 1086 (1995).

${ }^{3}$ T. Honda, K. Yanashima, F. Koyama, H. Kukimoto, and K. Iga, Jpn. J. Appl. Phys. 33, 1211 (1994).

${ }^{4}$ A. Salokatve, K. Rakennus, P. Uusimaa, M. Pessa, T. Aherne, J. P. Doran, J. O'Gorman, and J. Hegarty, Appl. Phys. Lett. 67, 407 (1995).

${ }^{5}$ Y. Raffle, R. Kuszelewicz, R. Azoulay, G. Le Roux, J. C. Michel, L. Dugrand, and E. Toussaere, Appl. Phys. Lett. 63, 3479 (1993).

${ }^{6}$ N. Nakayama, S. Itoh, T. Ohata, K. Nakano, H. Okuyama, M. Ozawa, A. Ishibashi, M. Ikeda, and Y. Mori, Electron. Lett. 29, 1488 (1993).

${ }^{7}$ A. Salokatve, H. Jeon, J. Ding, M. Hovinen, A. V. Nurmikko, D. C. Grillo, L. He, J. Han, Y. Fan, M. Ringle, and R. L. Gunshor, Electron. Lett. 29, 2192 (1993).

${ }^{8}$ M. Ukita, H. Okuyama, M. Ozawa, A. Ishibashi, K. Akimoto, and Y. Mori, Appl. Phys. Lett. 63, 2082 (1993).

${ }^{9}$ D. C. Grillo, M. D. Ringle, G. C. Hua, J. Han, and R. L. Gunshor, J. Vac. Sci. Technol. B 13, 720 (1995).

${ }^{10}$ J. D. Walker, K. Malloy, S. Wang, and J. S. Smith, Appl. Phys. Lett. 56, 2493 (1990) 